\title{
NONCOMMUTATIVE GEOMETRIES: AN OVERVIEW
}

\author{
CLAUDIO PERINI \\ Dipartimento di Matematica, Università degli Studi Roma Tre, \\ Roma I-00146, Italy \\ Received 15 Jenuary 2008 \\ Revised 15 jenuary 2008
}

\begin{abstract}
I make a very introductory overview of noncommutative geometries, focusing on the DFR model for Minkowski space; in this model the noncommutativity, or "fuzziness", of spacetime events emerges at semiclassical level putting together the Heisenberg principle with general relativity.
\end{abstract}

Keywords: noncommutative geometry; DFR model

PACS numbers: 02.40.Gh, 04.60.-m

\section{Introduction}

Spacetime at large scales is mathematically described by a pseudo-Riemannian manifold; spacetime curvature represents the gravitational field. It is generally believed that at the Planck scale spacetime becomes fuzzy, due to his very quantum nature; so gravity pass from the classical to the quantum regime. Why does the concept of quantum spacetime emerge? For aesthetic reasons: electroweak and strong forces are mediated by quantum fields, and the dream of every theoretical phisicist is to unify in a unique framework all the interactions, included gravity. For physical reasons: black holes are singular solutions to the Einstein equations, and taking into account the quantum nature of spacetime may cure those singularities; furthermore, Planck-scale effects generated by the quantum nature of spacetime may cure also the singularities arising in quantum field theories, in particular the nonrenormalizable ones. The main quantum gravity theories are loop quantum gravity (a canonical quantization of GR), string theory and noncommutative geometries.

In mathematics, geometrical objects can be often described in a purely algebraic way. For exemple, vector fields on manifolds are precisely the derivations of the algebra of continous functions on the manifold, and there are analogous definitions for general tensor fields, metric tensors, connections, etc. Switching from commutative to noncommutative algebras permits to define the noncommutative geometrical objects, which could be thought as objects on a noncommutative manifold. 
The mathematical task of ordinary quantum mechanics is to quantize the classical phase space (a sympletic manifold); the functions on the phase space, called observables, form a commutative algebra. So a way to quantize the phase space is to pass somehow to a noncommutative algebra, for example deforming the product to a noncommutative one. This procedure is called deformation quantization ${ }^{3,4}$.

Connes geometry ${ }^{2}$, instead, is a spectral characterization of Riemann compact manifolds. In fact these can be completely described by means of the eigenvalues of the so-called Dirac operator; generalizing this operator in the context of noncommutative algebras permits to define a noncommutative Riemannian manifold. Unfortunately Connes geometry does not work neither for Lorentzian nor for noncompact manifolds, just the physically interesting cases. However, some quantum models for Minkowski spacetime have been formulated. One of them is the DFR model, that furthermore is physically motivated.

\section{DFR model}

In ${ }^{1}$ Doplicher, Fredenhagen and Roberts suggested a quantum spacetime model motivated by operational arguments: putting together the uncertainty principle with GR gives semiclassical uncertainties on spacetime events localization. Imagine a localization experiment: we want to measure the position and time at which some event happens with precision $\Delta a$; according to the Heisenberg principle, an energy of order $1 / \Delta a$ must be transferred. We understand that there is a limit on the precision, because below some critical value a black hole is generated and the localization looses its operational meaning. Writing the conditions for no-formation of black holes, some limitations on spacetime coordinates arise:

$$
\begin{aligned}
& \Delta x_{0} \sum_{i=1}^{3} \Delta x_{i} \geq \lambda_{P}^{2} \\
& \sum_{i<j} \Delta x_{i} \Delta x_{j} \geq \lambda_{P}^{2}
\end{aligned}
$$

Now we may suppose that those semiclassical relations are a manifestation of the quantum nature of spacetime itself, and we may promote them to quantum mechanical uncertainties. In particular, coordinates will be noncommuting operators, and it is such noncommutativity that generates the uncertainties. In order to construct a quantum model of spacetime we can follow the path of ordinary quantum mechanics. In QM we search for:

1) commutation rules that generate the Heisenberg principle: $[p, x]=-i \hbar$;

2) concrete operators satisfying the commutation rules;

3) quantization of classical observables (not just position and momentum, but functions of both); geometrically, this problem is related to the quantization of the phase space.

In a similar way, in concommutative geometries spacetime coordinates do not commute and so a possible path to follow is to find: 
1) commutation rules that generate the uncertainties (1); these turn out to be the following:

$$
\begin{aligned}
& {\left[q_{\mu}, q_{\nu}\right]=i Q_{\mu \nu} \quad\left[q_{\mu}, Q_{\nu \rho}\right]=0} \\
& Q_{\mu \nu} Q^{\mu \nu}=0 \quad \frac{1}{16}\left(Q_{\mu \nu} * Q^{\mu \nu}\right)=1 ;
\end{aligned}
$$

2) concrete operators for which the previous commutation rules hold;

3) quantization of Minkowski spacetime, which is made indirectly by quantizing the classical functions.

\section{Mathematical background}

Classical observables are functions on some classical space; they form some kind of commutative algebra. Passing to noncommutative algebras gives a possible concept of quantum space. More specifically, we want to quantize topological spaces, and the structures stricly related to them are $\mathrm{C}^{*}$-algebras, as we shall see. So recall that a Banach algebra is an algebra (vector space endowed with moltiplication) endowed with a norm, respect to which the induced metric is complete. Banach algebras are the natural setup for spectral theory, and also for generalizing most of results of complex analysis. We will need a little more complicated structure, i.e. we endow the Banach algebra with the "star" operation *, also called adjoint operation, a unary operator with the same properties of adjoint operation for operators on Hilbert spaces. The resulting structure is called Banach *-algebra. $\mathrm{C}^{*}$-algebras are simply Banach ${ }^{*}$-algebras where the norm is such that

$$
\left\|x^{*} x\right\|=\|x\|^{2} . \quad\left(\mathrm{C}^{*} \text { identity }\right)
$$

Examples of $\mathrm{C}^{*}$-algeras are the set of complex matrices and the set of continuous operators on Hilbert spaces.

Commutative $\mathrm{C}^{*}$-algebras are completely characterized by Gel'fand theorem. Gel'fand theorem states that a given commutative $\mathrm{C}^{*}$-algebra is isomorphic to one of the following, depending on whether the algebra contains the unity or not:

1) $\mathscr{C}(X)$ : the $\mathrm{C}^{*}$-algebra of continuous complex functions on some locally compact, compact Hausdorff space $X$, with "star" operation given by complex conjugation and the norm of uniform convergence (the "sup" norm).

2) $\mathscr{C}_{0}(X)$ : the $\mathrm{C}^{*}$-algebra of continuous complex functions on some locally compact, non compact, Hausdorff space $X$, with the same "star" and norm.

Also for noncommutative $\mathrm{C}^{*}$-algebras there is a complete characterizations in terms of operators on some Hilber space; the proof is based on the GNS (Gel'fandNajmark-Segal) construction. By Gel'fand theorem, classical topologial spaces are mathematically equivalent to commutative $\mathrm{C}^{*}$-algebras, so the paradigma of noncommutative topology is to define quantum topological spaces as noncommutative $\mathrm{C}^{*}$-algebras; the elements of a $\mathrm{C}^{*}$-algebra can be interpreted as the "functions" on a quantum space. A comprehensive introduction to $\mathrm{C}^{*}$-algebras theory can be found in ${ }^{5}$. 


\section{DFR quantum spacetime}

As mentioned before, once written the commutation rules, the remaining steps to construct a quantum Minkowski space are two. First, one finds an explicit realization of the relations as operators on a Hilbert space; actually, DFR found a covariant realization (the Poincaré group is represented by unitary operators). Second, one finds the algebra generated by those operators, constituted by "functions" of the quantum coordinates, actually a noncommutative $\mathrm{C}^{*}$-algebra; explicitly this $\mathrm{C}^{*}$ algebra is $\mathscr{C}(\Sigma, \mathscr{K})$, i.e. the continous functions from a certain topological space $\Sigma$ to the set of compact operators on a separable Hilbert space, with the product and adjoint operations defined in a suitable way. Though it may appear a complicated object, this algebra is a little generalization of the noncommutative algebra of observables arising in ordinary quantum mechanics. Remarkably the Poincaré group is a symmetry of the DFR algebra. Recall that a symmetry of a topological space is a group acting as an homeomorphism on the space; at $\mathrm{C}^{*}$-algebra level it means that the group acts as an automorphism on the algebra. The latter characterization permits to define symmetries also for noncommutative topological spaces. Coming back to DFR model, the Poincaré group is a symmetry in this sense, and it acts as expected on the quantum coordinates $q^{\mu}$ :

$$
q^{\mu} \longmapsto \Lambda_{\nu}^{\mu} q^{\nu}+a^{\mu} .
$$

For this reason the DFR model is a quantum model for Minkowski space, where the symmetries are the same as the classical ones. The approach based on Hopf algebras ${ }^{6}$, instead, aims to quantum deform the classical symmetries.

\section{Outlook}

The way of quantizing general spacetimes seems not very clear, but a suitable generalization of the concepts exposed previously may give an answer. The missing ingredient is perhaps a deeper understanding of spacetime itself; LQG is an insight in this direction ${ }^{7}$. Noncommutative spacetimes could be structures emerging from a more fundamental theory of gravity.

\section{References}

1. S. Doplicher, K. Fredenhagen, J. E. Roberts, Commun.Math.Phys. 172 (1995) 187-220

2. A. Connes, Noncommutative Geometry, Academic Press, San Diego

3. F. Bayen, M. Flato, C. Fronsdal, A. Lichnerowicz, and D. Sternheimer, Lett. Math. Phys. 1 (1977), 521-530

4. F. Bayen, M. Flato, C. Fronsdal, A. Lichnerowicz, and D. Sternheimer, Ann. Phys. 111 (1978), 61-151

5. O. Bratteli, D.W. Robinson, Operator algebras and quantum statistical mechanics, vol. I, Springer, 1987

6. A. Agostini, G. Amelino-Camelia, F. D'Andrea, Int.J.Mod.Phys. A19 (2004) 5187-5220

7. C. Rovelli, Quantum Gravity, Cambridge University Press, 2004 